\title{
COMPARISON OF CURRENT SCREENING FOR PREVALENCE OF DEPRESSION AND ANXIETY WITH STANDARDIZED SCREENING TOOLS I PATIENT HEALTH QUESTIONNAIRE-9 AND GENERALISED ANXIETY DISORDER-7 AMONG ADULT INPATIENTS AT SHAUKAT KHANUM MEMORIAL CANCER HOSPITAL AND RESEARCH CENTRE
}

\author{
Suhaib Bin Bilal Hafi, Nasira Hayat, Nosheen Qureshi, Tanzila Tanzeem, Sheheryar Jovindah, \\ Haroon Hafeez \\ Department of Internal Medicine, Shaukat Khanum Memorial Cancer Hospital and Research Centre, Lahore, \\ Pakistan
}

Received: 12 December 2017 / Accepted: 22 November 2017

\begin{abstract}
Purpose: Screening for depression and anxiety for inpatients at Shaukat Khanum Memorial Cancer Hospital and Research Centre is done by nursing team by asking patients two questions from a structured admission tool aimed toward depression and anxiety. We audited the use of the current method of screening for depression and anxiety against standard screening tools for depression and anxiety i.e., Patient Health Questionnaire (PHQ)-9 and Generalised Anxiety Disorder (GAD)-7. The aim was improvement of screening for depression and anxiety amongst cancer inpatients.

Methods: We used a cross-sectional method to observe if the current screening method was being completed on admission on an inpatient ward of 40 patients on one particular day. Second, we delivered PHQ-9 and GAD-7 as screening tools on the same inpatients on the same day to compare with the current screening method.

Results: Prevalence of depression was 54.5\% $(n=18)$ and that of generalized anxiety was $36.4 \%(n=12)$, regardless of severity, when using PHQ-9 and GAD-7. Comparatively, the current method of screening was able to pick up $16.6 \%$ $(n=3)$ of cases of depression and $16.6 \%(n=2)$ of cases of generalised anxiety.
\end{abstract}

Conclusion: We concluded that improvement was required in the screening process for depression and anxiety by devising a new screening tool specific to our cancer population.

Key words: Anxiety, cancer, depression, screening

\section{Introduction}

Psycho-oncology Liaison Service provides mental health assessment, diagnosis and treatment for patients under the care of Shaukat Khanum Memorial Cancer Hospital and Research Centre (SKMCH\&RC). Research indicates that psychiatric morbidity in the form of depressive and anxiety disorders is very common amongst patients with malignancies. Accordingly, there is a need for

Correspondence: Suhaib Bin Bilal Hafi, MRCPsych Psychooncology Liaison Service, Department of Internal Medicine, Shaukat Khanum Memorial Cancer Hospital and Research Centre, Lahore, Pakistan.

Email: suhaibbilal@skm.org.pk close liaison between oncologists and mental health professionals to improve the outcome of patients with various malignancies. ${ }^{[1]}$ Better screening of patients suffering from depression and anxiety will improve access to mental health services resulting in better treatment outcomes. At present, screening for depression and anxiety at SKMCH\&RC is done on admission to the ward by asking two questions, one aimed toward depression and the other toward anxiety. These questions are part of a structured admission tool completed by nursing staff.

The aim of our study was to audit the use of the current screening method and observe if it was being completed on admission for each inpatient. 


\section{Methods}

\section{Standards used}

Screening method at SKMCH\&RC was that all inpatients should receive two questions from the admission document and if they answer yes to any of them, referral to Psycho-oncology Liaison Service was automatically generated by the Hospital Information System (HIS). This screening method has been in place for several years at SKMCH\&RC. There is some evidence that amongst the ultrashort measures (i.e., those containing one-four items), the Combined Depression Questions performed best in patients receiving palliative care; ${ }^{[2]}$ however, there is no significant evidence base for the method of screening question being used at SKMCH\&RC.

We compared this screening method with standard screening questionnaires i.e., Patient Health Questionnaire (PHQ)-9 and Generalised Anxiety Disorder (GAD)-7. ${ }^{[3]}$ These questionnaires have been used in other similar studies on similar populations. ${ }^{[1]}$ Another study has shown that PHQ - anxiety and depression scale (ADS) (which combines PHQ-9 and GAD-7) may be a reliable and valid composite measure of depression and anxiety. ${ }^{[4]}$ We used Urdu translation of PHQ-9 and GAD-7 which has been translated by the developers of the tool. Another study has reported that the Urdu version of the PHQ-9 has acceptable psychometric properties to screen and detect major depression in patients with coronary artery disease. ${ }^{[5]}$ It is an easy to use patient-administered questionnaire for screening, diagnosing and monitoring depression. It has nine questions based on DSM-V criteria for diagnosis of depression. Total score for the nine questions is 27 , 3 points for each question. Cutoff values for PHQ-9 are as follows:

- 0-4 No evidence of depression

- 5-9 Mild depression

- 10-14 Moderate depression

- 15-19 Moderately severe depression

- 20-27 Severe depression.

We used Urdu translation of GAD-7, which is an easy to use patient-administered questionnaire for screening and severity measure for generalised anxiety disorder. It has seven questions based on criteria for diagnosis of generalised anxiety disorder. Total score for seven questions is 21,3 points for each question. Cutoff values for GAD-7 are as follows:

- 0-4 No evidence of anxiety

- 5-9 Mild anxiety

- 10-14 Moderate anxiety

- 15-21 Severe anxiety.

No permission was required to reproduce, translate, display or distribute PHQ-9 and GAD-7.

\section{Patient selection}

We selected all inpatients on one cancer inpatient ward at SKMCH\&RC on one particular day i.e., $14^{\text {th }}$ November, 2017. There were 40 patients on the ward which were all included in the study. Verbal consent was obtained from patients at the time of distribution of the screening tool.

\section{Study method}

Initially, we audited the process of screening already in place i.e., two questions in the nursing admission document, by looking at patient admission nursing assessment for all inpatients in the ward, on HIS and observed if they had been completed.

The two questions already in place were as follows:

1. Do you feel stressed? Anxious? (feeling tense, muscle tension and sweating most of the time in the past 2 weeks)

2. Do you feel depressed? (Empty mood, loss of interest, helpless and disturbed sleep. At least one of these most of time for 2 weeks).

We then distributed the PHQ-9 and GAD-7 questionnaire to the same inpatients in the ward at SKMCH\&RC. Where patients were uneducated and needed help, the team conducting the project helped patients in completing the questionnaire. We also collected additional demographic details i.e., age, sex, diagnosis, marital status, employment status and education status.

\section{Statistics}

The data were collected by the team conducting the audit and analysed using IBM SPSS Statistics 20.

\section{Results}

The total number of patients on the inpatient ward was 40 . Questionnaires were completed on 33 of these patients. In 
two patients, questionnaire could not be completed due to language barrier, and in five patients, it could not be completed as they were suffering from delirium.

As for the results of the initial audit of whether the current method of screening was completed, 97.5\% $(n=39)$ of patients had the questions completed on admission. There was only one patient for whom this screening was not completed. The referral to Psycho-oncology Liaison Service was automatically generated by the system if answer was yes to any of the two questions.

In a total of 33 patient, where questionnaires were completed, $45.5 \%(n=15)$ had no evidence of depression picked by PHQ9. There were $27.3 \%(n=9)$ of cases of mild depression, $15.2 \%(n=5)$ of cases of moderate depression and there were $12.1 \%(n=4)$ of cases of moderately severe depression. The prevalence of depression regardless of the level of severity when screened by delivering PHQ-9 was $54.5 \%(n=18)$.

In a total of 33 patients, $63.6 \%(n=21)$ of patients did not have anxiety as measured by GAD-7. 27.3\% $(n=9)$ had mild anxiety, 3.0\% $(n=1)$ had moderate anxiety and $6.1 \%(n=2)$ had severe anxiety. The prevalence of anxiety irrespective of the level of severity when screened by delivering GAD-7 was $36.4 \%(n=12)$.

In a total of 33 patient, $45.5 \%(n=15)$ had no evidence of depression picked by PHQ9, of which three were referred to liaison team as identified by the current screening tool. There were $27.3 \%(n=9)$ of cases of mild depression, $15.2 \%(n=5)$ of cases of moderate depression and $12.1 \%(n=4)$ of cases of moderately severe depression as screened by PHQ-9. Comparatively, one case of each level of severity was picked up by the current method and referred to psycho-oncology liaison team. Hence, out of a potential $54.5 \%(n=18)$ referrals, the current method of screening was able to pick up $16.6 \%(n=3)$ referrals.

In a total of 33 patients, $63.6 \%(n=21)$ had no evidence of anxiety picked by GAD-7 and none of these were referred to liaison team. $27.3 \%(n=9)$ of patients had mild anxiety, one patient had moderate level of anxiety and two patients had severe level of anxiety. The current method of screening was able to pick up one case of mild anxiety and one case of severe anxiety disorder. Hence, of a potential $36.4 \%(n=12)$ referrals, the current method of screening was able to pick up $16.6 \%(n=2)$ referrals [Figures 1-5].

\section{Demographics}

Education and marriage status were available for 36 patients. $20 \%(n=8)$ were uneducated, $32.5 \%(n=13)$ were educated from primary to matric and $37.5 \%(n=15)$ were from intermediate to masters. $62.5 \%(n=25)$ were married and $27.5 \%(n=11)$ were unmarried. Of the 37 patients, where data for employment were available, $57.5 \%(n=23)$ were unemployed. There was a wide range of diagnosis in the patient group. Most frequent were CA breast which was diagnosed in $12.5 \%(n=5)$ of cases and pre-B ALL in $10 \%(n=4)$ of patients. There was no significant association of any particular type of cancer in these patients with diagnosis of depression or generalised anxiety disorder. The results showed that generalised anxiety disorder was significantly associated with the age group of 18-52 years. There were no other significant associations between gender, employment status and marital status with depression or generalised anxiety disorder.

\section{Discussion}

The initial audit showed that $97.5 \%$ of patients had the initial screening questions completed for depression and anxiety where it had not been completed in one case. We were not aware of the reason for it not being completed in one case as there was no such documentation on admission.

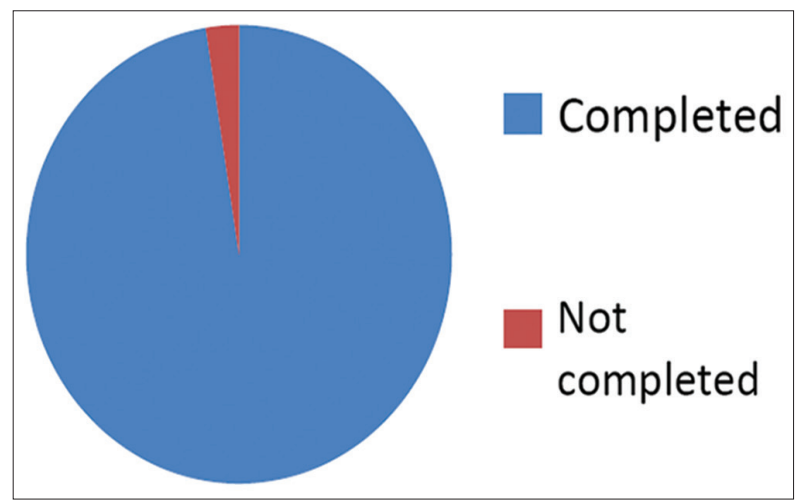

Figure 1: Current screening questions for depression and anxiety 
When we used PHQ-9 and GAD-7 on the same patients, prevalence of depression was $54.5 \%(n=18)$ and that of generalized anxiety was $36.4 \%(n=12)$ regardless of severity. The current method of screening was able to pick up $16.6 \%(n=3)$ of cases of depression and $16.6 \%$ $(n=2)$ of cases of generalised anxiety when compared with screening through PHQ-9 and GAD-7, respectively. The results of screening from the current method were not adequate even when different categories of depression were considered separately showing that rate of picking

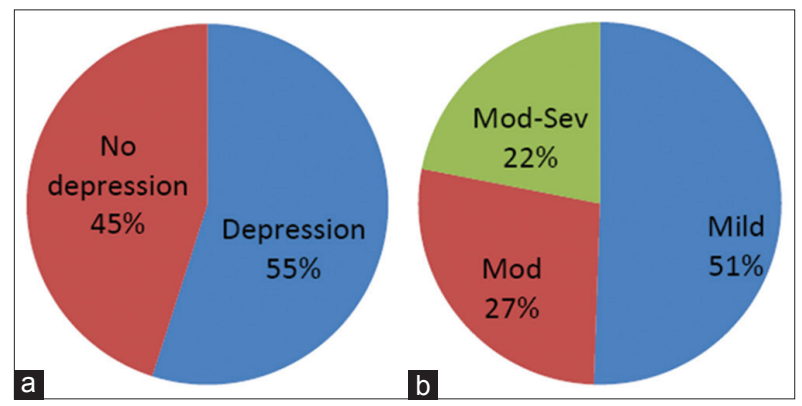

Figure 2: Prevalence of depression. (a) Patient health questionnaire-9, (b) Severity of depression

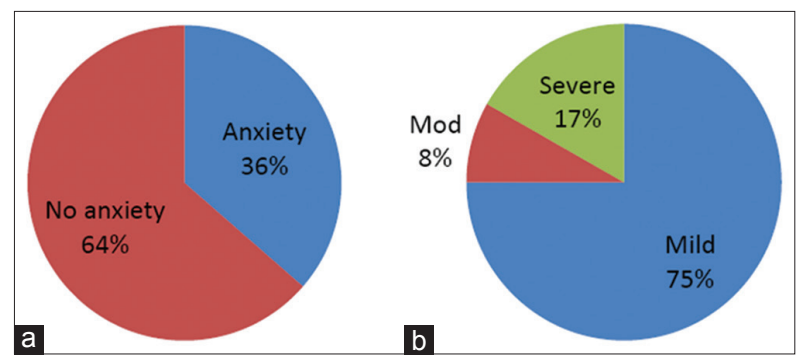

Figure 3: Prevalence of generalised anxiety disorder. (a) Generalised anxiety disorder-7, (b) Severity of anxiety depressive or anxiety symptoms was low across the spectrum of severity. It is important that the screening tool should be able to pick up cases across the level of severity as according to NICE guidance; ${ }^{[6]}$ all patients with mild depressive symptoms should be assessed by psychologist and identified for suitable psychological therapy. Patients with mild-to-moderate and severe symptoms should see a psychiatrist along with assessment from a psychologist and be considered for the need for psychotropic medication if indicated.

In our view, the reasons for low sensitivity of the current method were the fact that the screening questions were in English which require translation into Urdu by the staff member completing the questionnaire. Translating mental health symptoms into Urdu could be a difficult task specifically if the nursing staff was not trained to ask those specific questions. There was training put in place for staff members to ask these questions when this method was applied in the beginning; however, there had not been any audits to observe the application and success of this training. Furthermore, in our view, the questions asked for anxiety in the current method were very non-specific such as sweating and muscle tension. Furthermore, the questions for detecting depression were vague and non-specific such as empty mood and disturbed sleep.

Screening identifies patients with persistent depressive and anxiety symptoms and increases access to clinical assessment and support. ${ }^{[7]}$ However, time constraints of health professionals and insufficient knowledge about the appropriate screening tool may partially account for

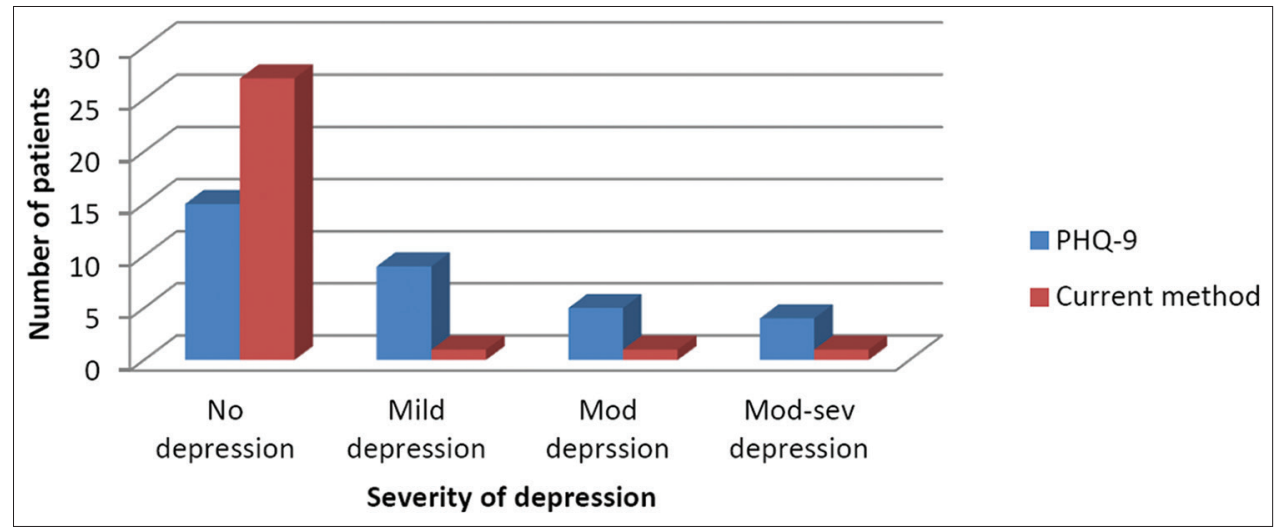

Figure 4: Patient health questionnaire-9 versus the current method of screening depression result 


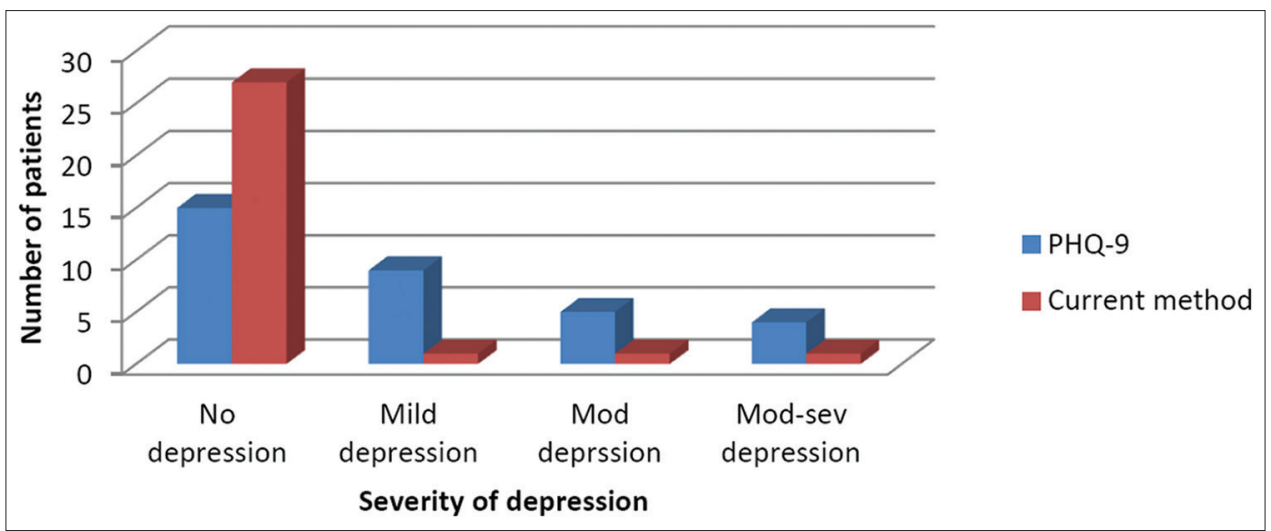

Figure 5: Generalised anxiety disorder-7 versus the current method of screening for anxiety result

the infrequent use of high-quality screening instruments in cancer care settings. The widely acknowledged shortage of professional staff for treatment followthrough suggests a need for screening tools with high sensitivity and high specificity that ensure that all patients in need of psychological support are identified. Routine use of longer tools, particularly their scoring and interpretation, places more of a burden on staff time. However, the availability of touch screen computerbased assessments can eliminate this disadvantage because the computer program can automatically score the assessment tool and generate a report. ${ }^{[2]}$ Most of the screening measures that have been validated for the use in cancer patients have between five and 20 items. Systematic review by Vodermaier concluded that the most extensive validation existed for the Hamilton ADS (HADS), and this was the case across disease types and stages as well as across languages and cultures. The scale has been extensively tested against criterion standards. ${ }^{[2]}$

The limitations of our study were that it was done on a relatively small patient group and targeted only the inpatient population. We have made considerations to include outpatient population also in future studies of screening for depression and anxiety to make the results more generalisable.

We concluded that the current screening method in place at SKMCH\&RC had low sensitivity of picking up depression and anxiety across all levels of severity when compared against PHQ-9 and GAD-7. There is room for improvement when it comes to screening for depression and anxiety in the inpatient cancer population. There are other indigenous screening methods in place in Pakistan such as AKUADS, ${ }^{[8]}$ which has been widely researched; however, it is a long screening tool with 25 questions which are not specific to cancer patients and would be difficult to administer to our population due to time constraints. There is, therefore, need for devising a new method of screening for depression and anxiety which is suited to this specific patient group. Patient-administered questionnaire such as HADS (Urdu Translation) uploaded on the computer system may be one way to address the practicality of administering such screening tools; however, this would require patients to be educated to a certain level to be able to use computer-based software. It would also take more staff time and require logistic support from IT department. We recognize that this may not be possible in the immediate future. Psycho-oncology liaison team at SKMCH\&RC will work on developing a new tool for screening of depression and anxiety which would be suitable to be applied in practice day to day on the local cancer population and in future test its validity and reliability when compared to standard tools such as HADS.

\section{Conflict of Interest}

The authors declare that they have no conflict of interest.

\section{References}

1. Shankar A, Dracham C, Ghoshal S, et al. Prevalence of depression and anxiety disorder in cancer patients: An institutional experience. Indian J Cancer 2016;53:432-4.

2. Vodermaier A, Linden W, Siu C. Screening for emotional distress in cancer patients: A systematic review of assessment 
instruments. J Natl Cancer Inst 2009;101:1464-88.

3. Patient Health Questionnaire Screeners. Available from: http://www.phqscreeners.com/select-screener/41. [Last accessed on 2017 Dec 01].

4. Kroenke K, Wu J, Yu W, et al. The patient health questionnaire anxiety and depression scale (PHQ-ADS): Initial validation in three clinical trials. Psychosom Med 2016; 78:716-27.

5. Gholizadeh L, Khan SA, Vahedi F, et al. Sensitivity and specificity of Urdu version of the PHQ-9 to screen depression in patients with coronary artery disease. Contemp Nurse 2017;53:75-81.
6. Nice Guidance. Depression in Adults: Recognition and Management; 2009. Available from: https://www.nice.org. uk/guidance/CG90. [Last accessed on 2017 Dec 01].

7. Thalén-Lindström A. Screening and Assessment of Distress, Anxiety, and Depression in Cancer Patients. Digital Comprehensive Summaries of Uppsala Dissertations from the Faculty of Medicine 1006. Uppsala: Acta Universitatis Upsaliensis; 2014. p. 75.

8. Ali BS, Reza H, Khan MM, et al. Development of an indigenous screening instrument in Pakistan: The Aga Khan university anxiety and depression scale. JPak MedAssoc 1998; 48:261-5. 